\title{
CATHETER ABLATION FOR ATRIAL FIBRILLATION ON UNINTERRUPTED DIRECT ORAL ANTICOAGULANTS: A SAFE APPROACH
}

Short Title: AF ablation on Uninterrupted DOACs

\author{
Sawhney V MPhiL MA ${ }^{1}$, Shaukat M MRCP ${ }^{1}$, Volkova E MRCP ${ }^{1}$, Jones N MRCP ${ }^{1}$, \\ Providencia R PhD ${ }^{1}$, Honarbakhsh S MRCP ${ }^{1}$, Dhillon G MRCP ${ }^{1}$, Chow A MD ${ }^{1}$, Lowe M \\ $\mathrm{PhD}^{1}$, Lambiase $\mathrm{PPhD}^{1}$, Dhinoja M FRCP ${ }^{1}$, Sporton S MD , Earley MJ MD ${ }^{1}$, Schilling RJ \\ MD ${ }^{1}$, Hunter RJ PhD ${ }^{1}$
}

1. Department of Arrhythmia Services, The Barts Heart Centre, St Bartholomew's Hospital, London, United Kingdom

*Corresponding Author: Vinit Sawhney

The Department of Arrhythmia services,

The Barts Heart Centre, St Bartholomew's Hospital,

London EC1A 7BE, UK.

Tel 4420376 58682, Fax 442034656483

Email: sawhneyvin@googlemail.com

This article has been accepted for publication and undergone full peer review but has not been through the copyediting, typesetting, pagination and proofreading process, which may lead to differences between this version and the Version of Record. Please cite this article as doi: $\underline{10.1111 / \text { pace. } 13370 \text {. }}$.

This article is protected by copyright. All rights reserved. 
Funding: cost-neutral study

\section{ABSTRACT}

Background: Current consensus guidelines suggest DOACs are interrupted periprocedurally for catheter ablation (CA) of AF. However, this may predispose patients to thromboembolic complications. This study investigates the safety of CA for AF on uninterrupted DOACs compared to uninterrupted warfarin.

Methods: Single centre, retrospective study of consecutive patients undergoing CA for AF. All patients were heparinised prior to trans-septal puncture with a target activated clotting time (ACT) of 300-350 seconds. Patients who had procedures performed on continuous DOAC were compared to those on continuous warfarin. Clinical, procedural data and complications occurring up to 3 months were analysed from a prospective registry with additional review of electronic health records.

Results: 1884 procedures were performed over 28 months: 761(609 patients) on uninterrupted warfarin and 1123(900 patients) on uninterrupted DOAC (rivaroxaban 64\%, apixaban $32 \%$ and dabigatran $4 \%$ ). There was no difference in the composite end point of death, thromboembolism or major bleeding complication( $2.2 \%$ vs $1.4 \%, p=0.20)$. There was no difference in the complications comprising this including tamponade, haematoma, pseudoaneurysm, transfusion( $p$-values $0.28,0.13,0.45$ and 0.36 ). There were no strokes, TIAs or other thromboembolic complications. There was no difference between groups in the proportion of tamponades requiring reversal of oral anticoagulation, the volume of blood lost, 
the proportion transfused, or the proportion drained percutaneously(p-values $0.50,0.51$, $0.36,0.38)$.

Conclusion: Catheter ablation for AF can be performed safely and effectively in patients anticoagulated with DOACs and heparinised with a therapeutic ACT. There is no increased risk of peri-procedural bleeding when compared to uninterrupted warfarin.

Keywords: Atrial fibrillation, catheter ablation, uninterrupted DOACs, uninterrupted warfarin

\section{INTRODUCTION}

Catheter ablation has emerged as an effective therapeutic option for patients with atrial fibrillation (AF). ${ }^{1}$ However, it is associated with low but serious risks including thromboembolic and bleeding complications. Peri-procedural management of anticoagulation is critical to minimise these risks. ${ }^{1}$

Vitamin $\mathrm{K}$ antagonists like warfarin have traditionally been used to prevent procedure related thromboembolism. Uninterrupted anticoagulation with warfarin during the periprocedural phase is associated with a lower thromboembolic and bleeding risk than cessation of warfarin and bridging with low molecular weight heparin (LMWH). ${ }^{2}$ However, obtaining optimal therapeutic international normalised ratio (INR) throughout the perioperative period remains a challenge. ${ }^{2}$ Sub-therapeutic INRs may increase thromboembolic risk whereas supra-therapeutic INRs may result in procedural cancellations or increased risk of vascular complications.

Direct Oral Anticoagulants (DOACs) are an attractive alternative in this setting as they provide various advantages over warfarin while having an equal or better safety profile than warfarin for systemic anticoagulation in non-valvular AF. ${ }^{3,4}$ However, clinical evidence 
for use of DOACs in the setting of AF ablation is limited. Current consensus guidelines advocate that DOACs be discontinued two days prior to catheter ablation (CA) due to increased bleeding risk. ${ }^{5}$ Available clinical evidence is predominantly from studies wherein the DOAC has been stopped prior to the procedure ${ }^{6-9}$ and hence potentially predisposing patients to the risk of thromboembolic complications. More recently, two randomised controlled trials (RCTs) have demonstrated that the use of uninterrupted dabigatran ${ }^{10}$ and rivaroxaban ${ }^{11}$ peri-procedurally for $\mathrm{AF}$ ablation is safe. However, the sample sizes in these studies were small for the purposes of studying infrequent complications. These were also selected patients in the controlled environment of randomised trials, with procedures being performed in world leading centres.

In this study we report our experience of catheter ablation for AF on uninterrupted DOACs in a large real world registry. We compared patients who underwent AF ablation on uninterrupted DOACs to those on uninterrupted warfarin in a case-control study to determine the safety and efficacy of both approaches.

\section{METHODS}

\section{Study design}

This was a retrospective, single-centre, case-control study evaluating the efficacy and safety of different anticoagulation regimens in the setting of CA for AF. Consecutive patients over 28 months were included. Patients on DOACs were compared to those anticoagulated with warfarin. Prior to the procedure, all patients gave written informed consent. All data presented were prospectively recorded in our departmental AF ablation registry.

\section{Study patients}

This article is protected by copyright. All rights reserved. 
All patients undergoing catheter ablation for $\mathrm{AF}$ and anticoagulated with either a DOAC (rivaroxaban, apixaban or dabigatran) or warfarin were included in the study. Edoxaban was not deliberately excluded but was not used for any patients during the study period. A transoesophageal echocardiogram (TOE) was performed prior to the procedure in all patients except those with paroxysmal AF who had been therapeutically anticoagulated for at least 3 weeks and were deemed not to be exceptionally high risk of stroke (e.g. hypertrophic cardiomyopathy, or previous stroke on anticoagulation). Those with left atrial appendage thrombus did not go on to have an ablation. Patients on uninterrupted DOACs were compared to those on uninterrupted warfarin.

\section{Anticoagulation regime}

An uninterrupted anticoagulation regime was adopted for all patients regardless of whether they were on a DOAC or warfarin. All patients were anticoagulated for at least 3 weeks prior to the ablation. For those taking warfarin, the target therapeutic INR was 2-3. INR was checked on a weekly basis prior to the procedure and on the day of the procedure.

Patients taking a twice-daily DOAC took their usual dose in the morning and the evening of the procedure. Patients taking a once daily DOAC were encouraged to take this in the evening for the week prior to the procedure, then took this the night before and the evening immediately after their procedure. However, patients still taking their dose in the morning continued this as usual.

Need for procedure cancellations because of anticoagulation issues (supratherapeutic INR on the day of the procedure, sub-therapeutic INR in the 3 previous weeks, or omitted DOAC doses) were compared. 
All patients were heparinised prior to trans-septal puncture with a target ACT of 300350 seconds. INR was re-checked for the warfarinised patients post-procedure. Oral anticoagulation was continued for at least 3 months post procedure in all patients - Figure 1 .

\section{Catheter ablation procedure}

Procedures were carried out either under intravenous moderate sedation or a general anaesthetic.

Vascular ultrasound was routinely used for obtaining venous access. Three punctures were performed for radiofrequency ablation and two for cryoballoon ablation. After achieving venous access patients were heparinised, with 5000 units of heparin given prior to trans-septal puncture and further boluses following trans-septal puncture aiming for an ACT of $300-350$ seconds.

Patients underwent standard ablation procedures as directed by the operator. These included pulmonary vein isolation with or without additional substrate modification. Ablation catheters used included cryo-balloon catheter (Arctic Front, Medtronic, Inc) and irrigated radiofrequency ablation catheters.

Post-procedure, heparin was reversed with protamine and a Femostop ${ }^{\mathrm{TM}}$ Gold (St.Jude Medical @, St.Paul, MN) applied to the site of vascular access. A transthoracic echocardiogram was performed routinely to look for evidence of pericardial effusion. The oral anticoagulant (whether DOAC or warfarin) was continued as normal following the procedure without omitting any doses.

\section{Management of bleeding complications}

This article is protected by copyright. All rights reserved. 
Acute procedural bleeding complications (mostly tamponade) were managed with a standardised protocol - Figure 2.

Data were collected on anticoagulant reversal at the time of tamponade, need of pericardiocentesis, amount of fluid drained, need of transfusion and need of sternotomy and surgical repair.

\section{Follow up}

Patients were kept in overnight and discharged home the following day. They were reviewed in clinic at 3 months post ablation. Additional review of medical notes and electronic health records was carried out to ensure capture of all complications.

\section{End points}

The primary end point of the study was a composite of death or any major bleeding or embolic complications over the follow-up period of at least 3 months. All complications were prospectively recorded in the nationally mandated institutional registry. All complications were reviewed to allow the application of a rigorous consensus definition compatible with guidelines $^{5,12}$. Complications were considered to be major if they resulted in permanent injury or death, required intervention for treatment or resulted in prolonged hospitalisation for $\geq 48$ hours. This included death, any thrombo-embolic event, tamponade and atriooesophageal fistula.

A stroke was defined as a sudden focal neurological deficit of presumed cerebrovascular aetiology lasting for $>24 \mathrm{~h}$, not due to another identifiable cause and confirmed by computed tomography or magnetic resonance imaging of the brain. If 
symptoms were short lasting $(<24 \mathrm{~h})$ and no evidence of necrosis was found on brain imaging, the event was considered to be a TIA. A systemic embolic event was defined as an abrupt vascular insufficiency associated with clinical or radiological evidence of arterial occlusion in the absence of another likely mechanism (e.g. atherosclerosis, instrumentation, or trauma). A pulmonary embolism was diagnosed when dyspnoea or other suggestive clinical presentation was accompanied by radiological confirmation of a new pulmonary perfusion or intra-luminal defect.

Bleeding complications considered to be major complications included cardiac tamponade, massive haemoptysis, haemothorax, retroperitoneal bleeding, or any other bleeding considered to be life threatening, necessitating intervention (including thrombin injection, placement of a drain or surgery), transfusion, or causing prolongation of hospitalisation or re-attendance to hospital following discharge. Bleeding complications such as groin haematomas not meeting these criteria were considered minor complications. Phrenic nerve palsy was considered a major complication if it persisted at the end of the procedure, but if transient and fully resolved by the end of the case was considered a minor complication.

\section{Statistical analyses}

Data were analysed on an intention-to-treat basis. All analyses were carried out using SAS version 9.3, statistical software. Continuous data were presented as mean \pm standard deviation or median (range) if not normally distributed. Categorical data were reported as a percentage. Continuous data were compared using unpaired t-test (if normally-distributed) and Mann-Whitney $U$ test if not normally-distributed. Categorical data were compared using chi-square test.

This article is protected by copyright. All rights reserved. 
Multivariate analyses were carried out using binary logistic regression. Two analyses were performed. Firstly the impact of DOAC usage on the incidence of bleeding complications was assessed in, both in a univariate model and in a multivariate model including persistent AF, cryoablation and gender as binary variables and age as a continuous variable. The second analysis split the cohort into those taking DOACs and those taking warfarin. The multivariate analysis was then repeated for each group with the same four other factors (age, gender, whether AF was persistent and type of ablation energy used i.e. cryo or radiofrequency) to see if these factors rendered patients at high risk of bleeding with either DOACs or warfarin. A p-value $<0.05$ was considered significant.

\section{RESULTS}

\section{Study patients}

1884 consecutive AF ablation procedures performed at a single institution over 28 months (May 2015 to Sep 2017) were included. 761 procedures (on 609 patients) were performed on uninterrupted warfarin (Warfarin group), and 1123 procedures (900 patients) on uninterrupted DOAC (DOAC group). Of the DOAC group $64 \%$ of the ablations were on uninterrupted rivaroxaban, $32 \%$ on apixaban and $4 \%$ on dabigatran. The two groups were well matched for clinical characteristics - Table 1.

\section{Procedural data}

All procedures were performed by a consultant electrophysiologist. Seventeen operators (consultants) at a single centre performed these procedures. All patients received intravenous unfractionated heparin after trans-septal puncture with a target ACT of $300-$

This article is protected by copyright. All rights reserved. 
350 seconds. There was no significant difference between the groups with respect to procedural data - Table 2.

During the study period a further 3 patients had their procedure cancelled due the presence of a left atrial appendage (LAA) thrombus. Two patients had been anticoagulated with rivaroxaban and the third with warfarin (with a therapeutic INR of $>2$ for over 3 months). There were no missed doses in any of these patients. All patients had persistent AF with a $\mathrm{CHA}_{2} \mathrm{DS}_{2}$-VASc score of 3,3 and 4 respectively.

\section{Study endpoints}

\section{$\underline{\text { Primary endpoint - Complications }}$}

There was no significant difference in the primary end point (a composite of death, thromboembolism or major bleeding complications) between the two groups (DOAC versus Warfarin) - Figure 3. Reported complications included death (1 versus $1 ; p=1$ ); cardiac tamponade, $n(\%), 19(1.7 \%)$ versus $8(1.1 \%), p=0.28$; groin haematoma, $n(\%), 3(0.3 \%)$ versus $6(0.8 \%), p=0.13$; and femoral pseudoaneurysm, $n(\%), 5(0.4 \%)$ versus $2(0.2 \%), p=$ 0.45. None of the patients had a thromboembolic complication (including transient ischaemic attack or stroke) peri-procedurally or during the three-month follow up period.

There were two reported deaths in the cohort: one patient in each group. The first patient on warfarin suffered an atrio-oesophageal fistula post ablation. The patient underwent surgery but had a prolonged hospital admission, which eventually lead to death 46 days post ablation. The second patient was in the DOAC group. This patient with arrhythmogenic ventricular cardiomyopathy had a cardiac tamponade four hours postprocedure and a percutaneous drain was inserted. This drained $450 \mathrm{ml}$ of blood, which did not re-accumulate. Other than transient hypotension he remained well throughout the 
tamponade. Despite having recovered from this he suffered a cardiac arrest the following day. The initial rhythm was ventricular tachycardia/fibrillation, which was electrically cardioverted to sinus rhythm. Cardiac output was not restored despite no re-accumulation of fluid in the pericardium or other clear cause. Postmortem showed that the drain, which was still in situ, was correctly placed and did not reveal any additional complication, any source of bleeding, or any cause of arrhythmia other than the underlying cardiomyopathy.

Of the tamponades that occurred, none were thought related to a steam pop, 16 of 27 were thought to be related to transseptal puncture $(11 / 19$ in the DOAC group compared to $5 / 8$ in the warfarin group; $p=1.00$ ), and 11 of 27 were thought not to be related to the transseptal puncture $(8 / 19$ in the DOAC group compared to $3 / 8$ in the warfarin group; $p=$ 1.00).

Two patients in the DOAC group (taking rivaroxaban) required sternotomy for tamponade with ongoing bleeding despite percutaneous drainage compared to zero in the warfarin group; $p=0.25$. The amount of pericardial fluid drained in the two cases was 1.8 and $3 \mathrm{~L}$ respectively. Rivaroxaban was reversed with Octaplex in both cases. The source of bleeding was a right atrial appendage perforation in one and left atrial appendage in the other. Both patients underwent surgical repair and did well post-operatively with a mean hospital stay of fourteen days. One of the two patients subsequently underwent a second ablation in six months time.

The majority of tamponades were managed with reversal of heparin but without reversal of the oral anticoagulant. The oral anticoagulant was reversed in 1 out of $8(12 \%)$ of cardiac tamponades on warfarin compared to 2 of $19(11 \%)$ of cardiac tamponades on a DOAC (both rivaroxaban).

Pericardial fluid drained was not significantly different in the two groups (DOAC versus warfarin; $710 \pm 748 \mathrm{ml}$ versus $532 \pm 113 \mathrm{ml} ; \mathrm{p}=0.51$ ). Only a small proportion of patients required blood transfusion post procedure $(n=3,0.3 \%$ in the DOAC group and $n=$ This article is protected by copyright. All rights reserved. 
$1,0.1 \%$ in the warfarin group; $p=0.36$ ). All of them had suffered blood loss as a result of cardiac tamponade.

Ongoing haemoptysis for four days post ablation was seen in one patient in the warfarin group. There was no evidence of pulmonary vein stenosis on imaging. This was attributed to interstitial lung disease due to amiodarone and recovered with conservative management alone.

\section{Multivariate analysis of factors impacting bleeding risk}

A univariate analysis did not show any significant association between the use of DOAC and bleeding events $(\mathrm{HR} 0.39, \mathrm{Cl} 0.09-1.73, \mathrm{p}=0.21)$. This was confirmed in a multivariate model, with no significant association between bleeding events and DOAC usage, age, gender, type of AF and ablation energy (cryoablation versus radiofrequency) (DOAC: HR 0.50, Cl 0.11-2.35, p = 0.38; Age: HR 1.05, Cl $0.99-1.11, p=0.10$; male gender: HR 2.07, Cl 0.63 - 6.86, $p=0.23$; Paroxysmal AF: HR 0.56, Cl $0.16-1.9, p=0.37$; Cryoablation HR $2.9, \mathrm{Cl} 0.77-11.30 \mathrm{p}=0.112)$.

Multivariate regression analyses were also carried out to assess the predictors of major bleeding complications in patients taking either DOACs or a vitamin $\mathrm{K}$ antagonist. There was no significant association between age, gender, type of AF (paroxysmal/persistent) and ablation energy (cryoablation versus radiofrequency) and bleeding events in those taking warfarin (Age: HR 1.09, Cl $0.89-1.32, p=0.340$; male gender: HR 1.01, Cl $0.05-19.21, p=0.99 ;$ Paroxysmal AF: HR $01.00 \mathrm{Cl} 0.06-16.85$, p=0.99, Cryoablation HR 0.55, Cl $0.03-9.79, \mathrm{p}=0.68$ ) or DOACs (Age: HR 1.04, Cl 0.99 1.11, $p=0.16$; male gender: HR 2.37, Cl $0.633-8.9, p=0.20$; Paroxysmal AF: HR 0.50, Cl $0.12-2.02, p=0.33$, Cryoablation HR 4.4, Cl $0.92-10.21, p=0.08$ ) groups. 


\section{Other major complications}

One patient in the DOAC group presented with a pharyngeal perforation two days post procedure. This was likely due to the TOE and was managed conservatively. Six patients in the DOAC group and one patient in the warfarin group had phrenic nerve palsy following cryoablation for paroxysmal AF DDOAC versus warfarin $n(\%) ; 6(0.5)$ versus $1(0.2)$, p-value 0.29 . Complete resolution in all but one, was seen on fluoroscopy at three and six months follow-up respectively (Table 3).

\section{Secondary endpoint - Procedural Success}

Acute ablation success (defined as restoration of sinus rhythm and confirmed pulmonary vein isolation) was similar in both groups \{DOAC versus warfarin; $n(\%) ; 587(98.35)$ and $590(98.8 \%) ; p=0.47\}$.

\section{Procedure cancellations}

No procedures in the DOAC group were cancelled due to anticoagulation issues. However, 23 patients who were anticoagulated with warfarin had their procedure cancelled due to an INR out of the therapeutic range (DOAC versus warfarin; 0 versus $3.02 \%, p<0.001$ ).

\section{DISCUSSION}

In the present study we report our experience of performing catheter ablation for AF on uninterrupted DOACs in a large unselected real world registry. In over a thousand patients ablated on uninterrupted DOACs this approach was found to be safe and effective with no This article is protected by copyright. All rights reserved. 
increased risk of peri-procedural bleeding or thromboembolism compared to uninterrupted warfarin. Moreover, there was no significant difference in the overall complication rate between the DOAC and warfarin group. There were no strokes or TIAs at all in either group with continuous oral anticoagulation, although a system of selective TOE did reveal a small number of patients with LAA thrombus, which might have resulted in systemic embolism if this had gone undetected. Vascular access related bleeding complications were very low $(<1 \%)$ with the use of continuous oral anticoagulation (DOAC or warfarin) in combination with vascular ultrasound for obtaining venous access and routine use of protamine and femostop post procedure. The greater cancellation rate due to anticoagulation issues in the warfarin group suggests that performing AF ablation on uninterrupted DOACs may have practical and economical advantages.

Although catheter ablation is a well-established and effective treatment option ${ }^{13,14}$ for patients with symptomatic $A F$, it is associated with a significant risk of peri- and post procedural complications. ${ }^{1,14}$ The increased risk of thromboembolic complications is likely related to the exacerbation of baseline pro-thrombotic state by catheters in the left atrium, endothelial denudation and tissue inflammation as a result of ablation. ${ }^{15}$ Effective anticoagulation is key to prevent the thromboembolic risk associated with AF ablation while balancing the associated bleeding risks. Traditionally Vitamin $\mathrm{K}$ antagonists like warfarin have been used to prevent pre- and peri-procedural thromboembolism and current consensus guidelines advocate the use of uninterrupted warfarin for AF ablation. ${ }^{5}$ However, INR levels often fluctuate during warfarin use, and may not be in the optimal therapeutic range in up to $50 \%$ of patients. ${ }^{16}$ Moreover, lower or higher INR levels on the day of ablation may increase the risk of complications. Recently, DOACs have been shown to offer important advantages over warfarin. These include rapid onset of action with shorter time required to achieve therapeutic anticoagulation, less interactions and no need for laboratory monitoring and ease of administration. However, clinical practice with the peri-procedural use of DOACs in AF ablation is inconsistent and the existing evidence comes from a small 
number of registry studies with small patient cohorts. Current EHRA guidelines suggest discontinuation of DOACs $24-48$ hrs prior to ablation. ${ }^{5,17}$

The preliminary data from registry studies on patients undergoing AF ablation on DOACs shows the safety and efficacy of this approach. However, in all of these studies DOACs were discontinued for $6-48$ hours prior to the procedure and re-started $6-24$ hours after haemostasis was achieved post ablation. ${ }^{9,10,18,19}$ In some studies bridging with LMWH was also used. This is therefore the largest registry examining AF ablation on DOACs and is also the only one in which DOACs were genuinely uninterrupted. Other registry studies have reported a significantly higher risk of bleeding with the use of a DOAC (dabigatran) peri-procedurally when compared to warfarin. ${ }^{6}$ This study reported a generally high risk of both thromboembolic complications and bleeding risk associated with AF ablation (dabigatran versus warfarin; 16 versus $6 \%$ and 6 versus $1 \%$ ). ${ }^{6}$ The higher bleeding risk was attributed to discontinuation of dabigatran on the morning of the procedure (and not earlier i.e. $24-48 \mathrm{hrs}$ ) and resuming it within 3 hours of haemostasis post-procedure. The unusually high tamponade rate was notable at $6 \%$ but unexplained.

Clinical experience and existing data with use of DOACs in catheter ablation of AF remains limited. ${ }^{20,21}$ Two recent RCTs have demonstrated the safety of performing AF ablation on uninterrupted DOACs: VENTURE AF with rivaroxaban and RE-CIRCUIT with dabigatran. ${ }^{10,17}$ VENTURE-AF was the first prospective randomised trial of uninterrupted rivaroxaban and vitamin $\mathrm{K}$ antagonists (VKA) in patients with non-valvular AF undergoing catheter ablation. With a primary end point of major bleeding events after catheter ablation, the use of uninterrupted rivaroxaban was feasible and comparable to those for uninterrupted VKA therapy. ${ }^{10}$ The RE-CIRCUIT trial investigated the safety of uninterrupted dabigatran versus VKA in patients undergoing AF ablation. The incidence of major bleeding was found to be lower in patients with uninterrupted dabigatran. ${ }^{17}$ 
However, these trials were under strict RCT conditions, had a smaller sample size than this registry and were limited to single DOACs. Although RCTs are the gold standard for clinical research, registry data is arguably complimentary in that patients may be less selected and a larger number of patients may be included with the potential to provide additional data regarding infrequent complications. The initial evidence provided by these RCTs is confirmed in our study representing real world registry data. The present study is the largest to date, to investigate the use of a variety of uninterrupted DOACs (pre-, peri- and post-procedural) in catheter ablation of AF and compare it to an appropriately matched control group on uninterrupted warfarin. The results of our study suggest that it is feasible to perform AF ablation on uninterrupted DOACs safely as the overall complication rate remained low and there was no significant difference in major and minor complications when compared to patients on uninterrupted warfarin.

There were no thromboembolic complications in either group with continuous oral anticoagulation and a system of selective TOE. There was no difference in the bleeding complications and in particular the tamponade rate. Although the overall rate of tamponades in this group was low, it is plausible that the use of TOE or ICE (Intra-cardiac echocardiography) guided transseptal punctures could have reduced this further in both groups. Furthermore, management of tamponades was similar in the two groups: most tamponades resolved with percutaneous drainage and reversal of heparin and did not require reversal of oral anticoagulation. The volumes of blood drained were similar in the two groups and transfusion rates low. Thoracotomy was required in two patients who were taking a DOAC: this was due to a perforation which required surgical closure and was unlikely to be related to the choice of oral anticoagulant, and the peri-operative course was uncomplicated. Both these patients were anticoagulated with rivaroxaban and heparinised with therapeutic ACTs. $\geq 1.8 \mathrm{~L}$ of blood was drained in both cases and they continued to bleed despite reversal of anticoagulation. Given the life threatening bleeding, reversal of the DOAC was required peri-operatively in both patients using Octaplex. Although limited data are available

This article is protected by copyright. All rights reserved. 
to guide clinical decision-making about the approach used to reverse the anticoagulant effects of DOACs; this is an important consideration in emergent and life threatening situations.

Multivariate analysis showed no suggestion of increased bleeding risk with DOACs. Furthermore, there was no subgroup identified as being at high risk of bleeding complications in either those taking DOACs or those taking vitamin $\mathrm{K}$ antagonists.

The current guidelines for AF ablation in patients taking DOACs, involves cessation of DOACs and bridging with LMWH. The data comparing bridging with $\mathrm{LMWH}$ to continuous oral anticoagulation previously derived from the field's experience with warfarin has suggested an increased rate of stroke with bridging LMWH. Moreover, bridging with LMWH is associated with potentially increased bleeding risks ${ }^{6}$ and is an expensive and cumbersome approach. There may therefore be a similar danger with this approach for DOACs.

We used a system of selective TOE to screen for LAA thrombus. Even with continuous oral anticoagulation (warfarin or DOAC) a small number of LAA thrombi were identified. The selective system of TOE described presumably identified all LAA thrombi as there were no strokes in the cohort. This suggests that TOE is not essential to rule this out in all patients, but that some system of screening ought to be considered (whether with TOE, intra-cardiac echo or CT left atrium).

\section{STUDY LIMITATIONS}

This is a retrospective, observational and non-randomised study. All procedures were performed at a single high volume arrhythmia centre with expertise in performing AF ablations. The outcomes may be influenced by operator experience.

This article is protected by copyright. All rights reserved. 
Although there was no signal for concern regarding any particular DOAC, given the low numbers of events it was not possible to assess the association of individual DOACs with outcomes in a multivariate model. Furthermore, no patients in this cohort were taking Edoxaban.

Despite the large number of patients included, only 2 patients required the reversal of anticoagulation with Octaplex (both for Rivaroxaban). It should be noted that this is not a specific reversal agent (reversal agents are not available for Factor Xa inhibitors at the time of writing). This study was therefore still underpowered to fully assess the management of serious bleeding complications, which require reversal of anticoagulation for DOACs.

\section{CONCLUSIONS}

This study shows that catheter ablation for atrial fibrillation can be performed safely and effectively in patients anticoagulated with uninterrupted DOACs in addition to systemic heparinisation (with therapeutic ACT). The overall complication rate including bleeding risk was not increased with this approach compared to uninterrupted warfarin. Taken together with the results of recent RCTs examining this practice, it may be appropriate to revise current guidelines, which recommend peri-procedural interruption of DOACs. Performing AF ablation on uninterrupted DOACs led to fewer cancellations than with uninterrupted warfarin suggesting this approach may also be more practical.

\section{ACKNOWLEDGEMENTS}

This study was facilitated by the Barts Health NHS Trust.

\section{COMPETING INTERESTS}

This article is protected by copyright. All rights reserved. 
RP has received speaker fees from Pfeizer; other authors report no relationships that could be construed as a conflict of interest.

\section{CONTRIBUTORSHIP}

VS: data collection, data analyses, manuscript draft and preparation; MS,EV,NJ: data collection; RP,SH,GD,AC,ML,PL,MD,SS,MJE,RJS: manuscript review; RH: study concept and design, supervised data collection and manuscript draft, manuscript review.

\section{REFERENCES}

1. Cappato R, Calkins H, Chen SA, Davies W, lesaka Y, Kalman J, Kim YH et al. Updated worldwide survey on the methods, efficacy, and safety of catheter ablation for human atrial fibrillation. Circ Arrhythm Electrophysiol. 2010;(3): 32-38.

2. DiBiase L, Burkhardt JD, Mohanty P, Sanchez J, Horton R, Gallinghouse GJ, Lakkireddy $D$ et al. Periprocedural stroke and management of major bleeding complications inpatients undergoing catheter ablation of atrial fibrillation: the impact of periprocedural therapeutic international normal-ized ratio. Circulation 2010; 121:2550-2556.

3. Connolly SJ, Ezekowitz MD, Yusuf S, Eikelboom J, Oldgren J, Parekh A, Pogue J et al Dabigatran versus warfarin in patients with atrial fibrillation. N Engl J Med 2009;361:1139_ 1151.

4. Patel MR, Mahaffey KW, Garg J, Pan G, Singer DE, Hacke W. Breithardt G et al. Rivaroxaban versus warfarin in nonvalvular atrial fibrillation. N Engl J Med 2011;365: 883891.

5. Calkins H, Kuck KH, Cappato R, Brugada J, Camm JA, Chen S, Crijns H et al. 2012 HRS/EHRA/ECAS Expert Consensus Statement on Catheter and Surgical Ablation of Atrial Fibrillation: Recommendations for patient selection, procedural techniques, patient management and follow-up, definitions, endpoints, and research trial design. Europace 2012;14: 528-606

This article is protected by copyright. All rights reserved. 
6. Lakkireddy D, Reddy YM, Di Biase L, Vanga SR, Santangeli P, Swarup V, Pimentel R et al. Feasibility and safety of dabigatran versus warfarin for periprocedural anticoagulation in patients undergoing radiofrequency ablation for atrial fibrillation: Results from a multicenter prospective registry. J Am Coll Cardiol. 2012;59:1168-1174.

7. Kaseno K, Naito S, Nakamura K, Sakamota T, Sasaki T, Tsukada N, Hayano M, et al. Efficacy and safety of periprocedural dabigatran in patients undergoing catheter ablation of atrial fibrillation. Circ J. 2012;76:2337-2342.

8. Snipelisky D, Kauffman C, Prussak K, Johns G, Venkatachalam K, Kusumoto F. A comparison of bleeding complications post-ablation between warfarin and dabigatran. $J$ Interv Card Electrophysiol. 2012;35:29-33.

9. Bassiouny M, Saliba W, Rickard J, Shao M, Sey A, Diab M, Martin DO et al. Use of dabigatran for periprocedural anticoagulation in patients undergoing catheter ablation for atrial fibrillation. Circ Arrhythm Electrophysiol. 2013;6:460-466.

10. Cappato R, Marchlinski FE, Hohnloser SH, Naccarelli GV, Xiang J, Wilber DJ. Ma CS et al. Uninterrupted rivaroxaban vs. uninterrupted vitamin $\mathrm{K}$ antagonists for catheter ablation in non-valvular atrial fibrillation. Eur Heart J 2015;38:1805-1811.

11. Calkins H, Willems S, Gerstenfeld EP, Verma A, Schilling R, Hohnloser SH, Okomura K et al. RE-CIRCUIT Investigators. Uninterrupted Dabigatran versus Warfarin for Ablation in Atrial Fibrillation. N Engl J Med. 2017;376:1627-1636.

12. Schulman S, Kearon C. Subcommittee on Control of Anticoagulation of the Scientific and Standardization Committee of the International Society on Thrombosis and Haemostasis. Definition of major bleeding in clinical investigations of antihemostatic medicinal products in non-surgical patients, J Thromb Haemostat. 2005;3:692-4.

13. Tilz RR, Rillig A, Thum AM, Arya A, Wohimuth P, Metzner A, Matthew S et al. Catheter ablation of long-standing persistent atrial fibrillation: 5-Year outcomes of the Hamburg Sequential Ablation Strategy. J Am Coll Cardiol 2012;60:1921-1929.

This article is protected by copyright. All rights reserved. 
14. Rillig A, Makimoto H, Wegner J, Linn T, Heeger C, Lemes C, Fink T et al. Six-year clinical outcomes after catheter ablation of atrial fibrillation in patients with impaired left ventricular function. J Cardiovasc Electrophysiol 2015;26:1169-1179

15. Viles-Gonzalez JF, Mehta D. Thromboembolic risk and anticoagulation strategies in patients undergoing catheter ablation for atrial fibrillation. Curr Cardiol Rep 2011;13:38-42.

16. Connolly SJ, Pogue J, Eikelboom J, Flaker G, Commerford P, Franzosi MG, Healey JS et al. Benefit of oral anticoagulant over antiplatelet therapy in atrial fibrillation depends on the quality of international normalized ratio control achieved by centers and countries as measured by time in therapeutic range. Circulation. 2008;118:2029-2037.

17. Heidbuchel $\mathrm{H}$, Verhamme $\mathrm{P}$, Alings $\mathrm{M}$, Antz $\mathrm{M}$, Diener HC, Hacke W, Oldgren $\mathrm{J}$ et al. Updated European Heart Rhythm Association Practical Guide on the use of non-vitamin K antagonist anticoagulants in patients with non-valvular atrial fibrillation. Europace 2015;17:1467-1507.

18. Riling A, Lin T, Plesman J, Heeger $\mathrm{CH}$, Lemes C, Metzner A, Mathew S et al. Apixaban, Rivaroxaban, and Dabigatran in Patients Undergoing Atrial Fibrillation Ablation. J Cardiovasc Electrophysiol. 2016;27:147-153.

19. Providencia R, Marijon E, Albenque JP, Combes S, Combes N, Jourda F, Hireche H et al..Rivaroxaban and dabigatran in patients undergoing catheter ablation of atrial fibrillation. Europace 2014;16:1137-1144.

20. Shin DG, Kim TH, Uhm JS, Kim JY, Joung B, Lee MH, Pak HN. Early Experience of Novel Oral Anticoagulants in Catheter Ablation for Atrial Fibrillation: Efficacy and Safety Comparison to Warfarin. Yonsei Med J. 2016;57:342-349.

21. Vallakati A, Sharma A, Madmani M, Reddy M, Kanmanthareddy A, Gunda S, Lakkireddy D et al. Efficacy and Safety of Novel Oral Anticoagulants for Atrial Fibrillation Ablation: An Updated Meta-Analysis. Cardiol Ther 2016;5:85-100 


\section{FIGURE LEGENDS}

\section{Figure 1: Anticoagulation Regime}

Anticoagulation regime (pre, peri and post procedural) for all patients undergoing catheter ablation for AF is shown here. DOAC: Non-vitamin K Oral Anticoagulants, INR: international normalized ratio, AF: atrial fibrillation, UFH: unfractionated heparin.

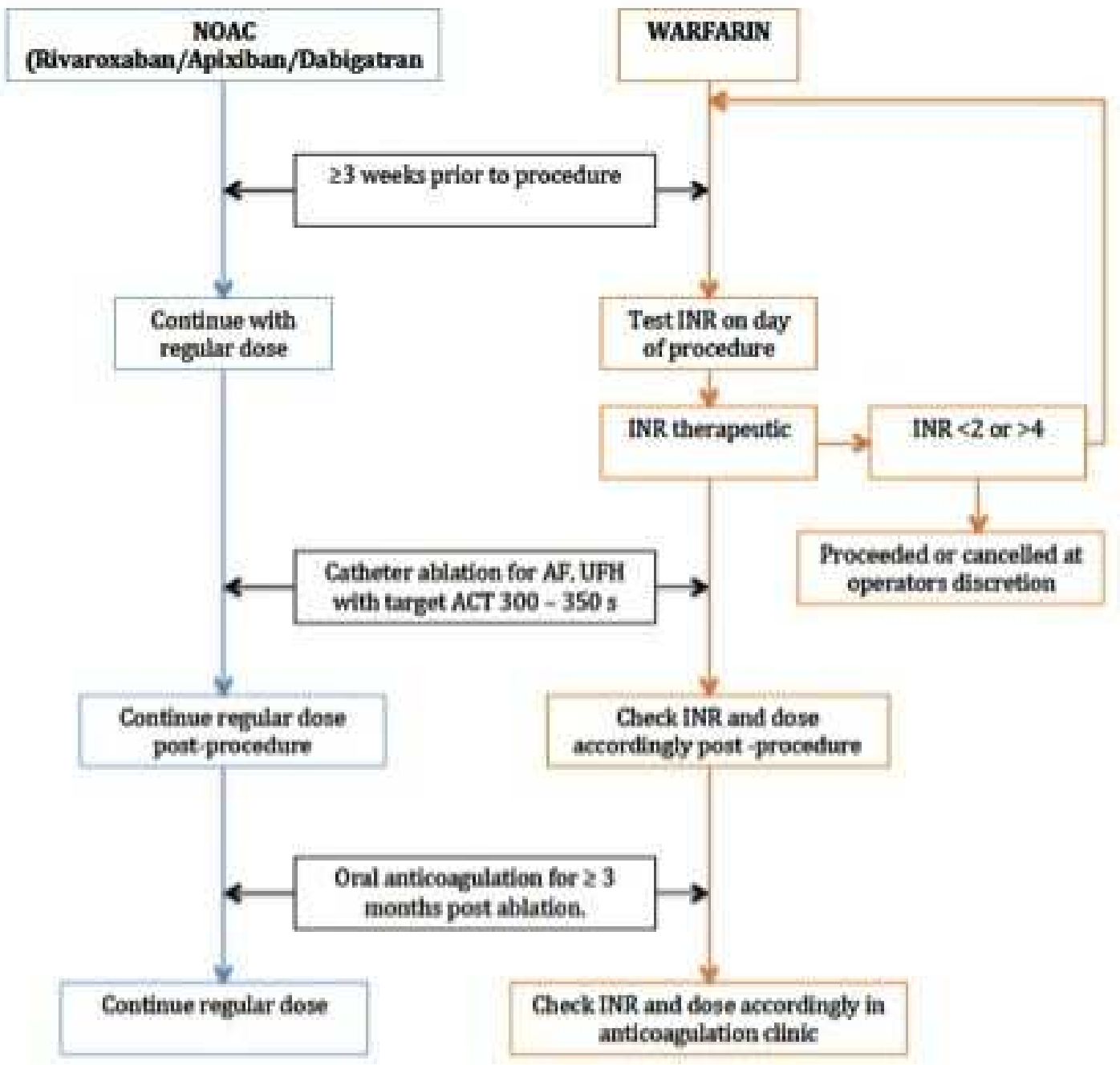

Figure 2: Protocol for Management of Cardiac Tamponade

Acute bleeding complication (cardiac tamponade) was managed based on a standardised protocol as detailed in this flowchart.

This article is protected by copyright. All rights reserved. 


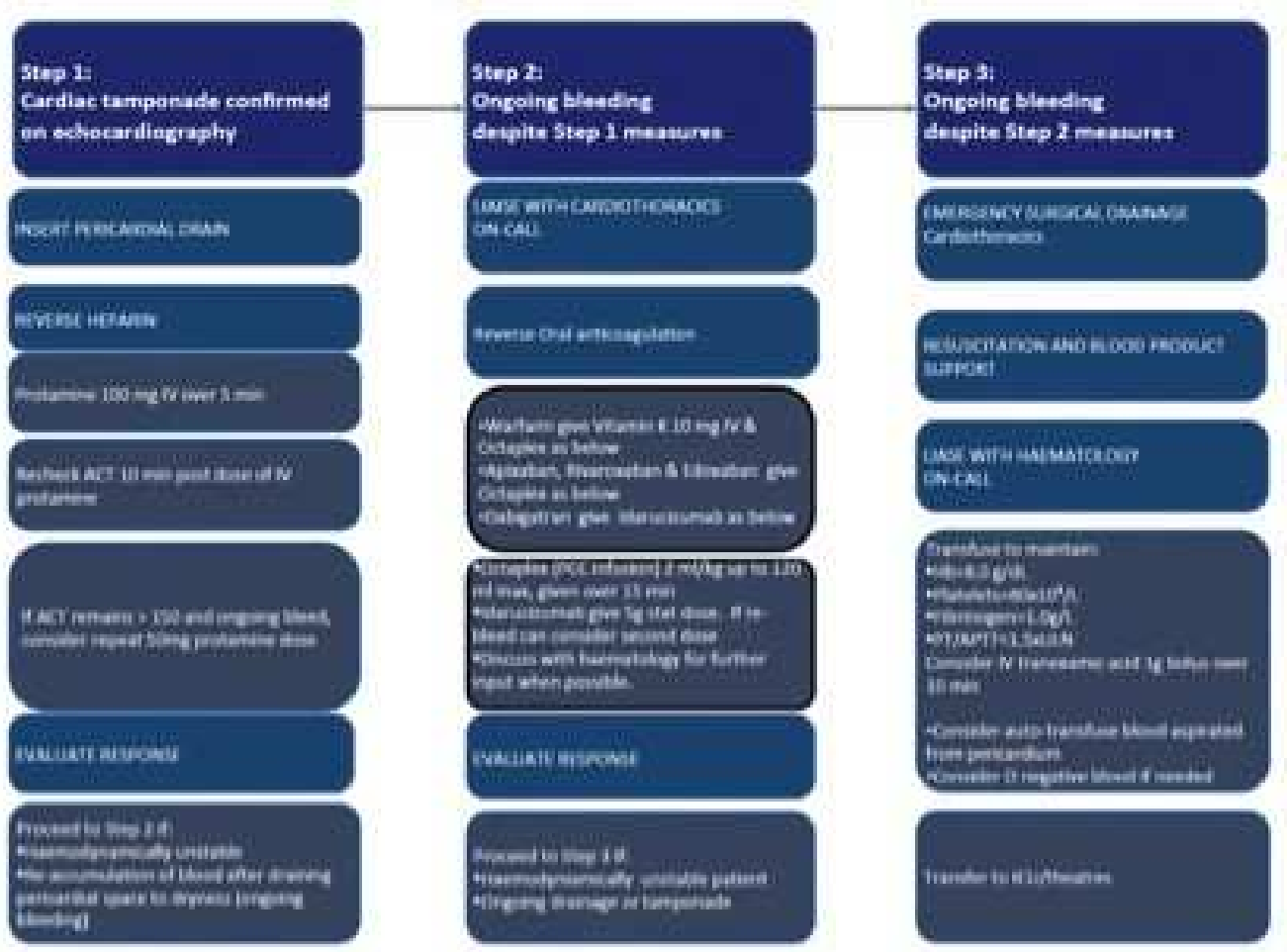

Figure 3: Primary End point (death, thromboembolism or major bleeding complications)DOAC versus Warfarin

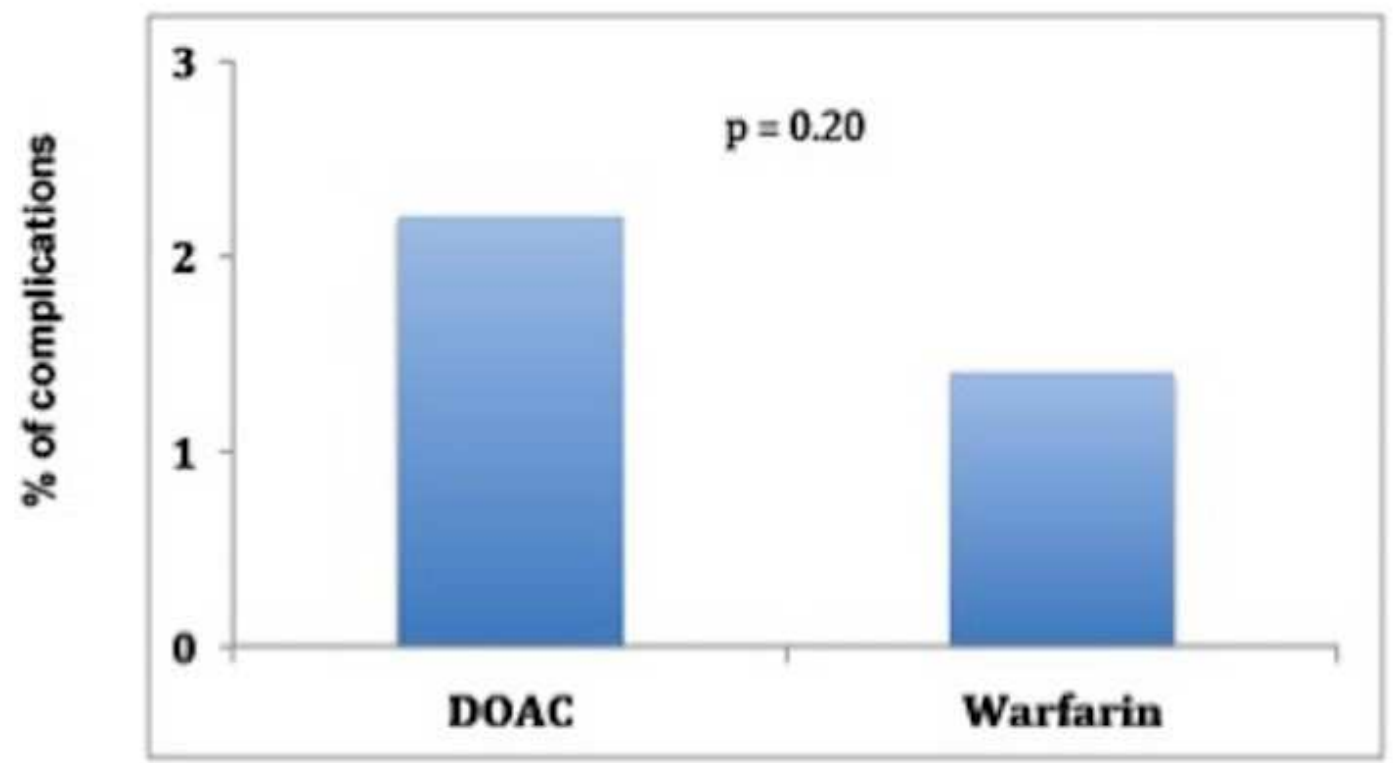

The complications comprising the primary end point in both DOAC and warfarin groups are shown here. There was no significant difference between the two groups.

This article is protected by copyright. All rights reserved. 


\begin{tabular}{|c|c|c|c|}
\hline Sex,M, $n(\%)$ & $613(55)$ & $393(52)$ & 0.20 \\
\hline Age, years, (mean $\pm S D)$ & $61 \pm 12$ & $62 \pm 14$ & 0.10 \\
\hline \multicolumn{4}{|l|}{$\begin{array}{l}\text { Background heart } \\
\text { disease }\end{array}$} \\
\hline Normal heart, $\mathrm{n}(\%)$ & $868(77)$ & $561(74)$ & 0.13 \\
\hline $\begin{array}{l}\text { Ischemic heart disease } \\
\mathrm{n}(\%)\end{array}$ & $61(5)$ & $51(7)$ & 0.07 \\
\hline $\begin{array}{l}\text { Dilated cardiomyopathy } \\
\mathrm{n}(\%)\end{array}$ & $94(8)$ & $64(8)$ & 1 \\
\hline Valvular, n(\%) & $32(3)$ & $30(4)$ & 0.24 \\
\hline Others, n(\%) & $68(6)$ & $55(7)$ & 0.38 \\
\hline \multicolumn{4}{|l|}{$A F, n(\%)$} \\
\hline Persistent & $504(45)$ & $332(44)$ & 0.66 \\
\hline Paroxysmal & $619(55)$ & $429(56)$ & 0.66 \\
\hline Hypertension, n(\%) & $721(64)$ & $479(63)$ & 0.65 \\
\hline Diabetes mellitus, $\mathrm{n}(\%)$ & $621(55)$ & $409(54)$ & 0.66 \\
\hline
\end{tabular}

Table 1: Clinical Characteristics. The clinical characteristics of the DOAC $(n=1123)$ and warfarin $(n=761)$ group are shown in this table. Data are reported as percentage or mean \pm SD. 


\begin{tabular}{|c|c|c|c|}
\hline \multicolumn{4}{|c|}{ Paroxysmal AF, n(\%) } \\
\hline De-novo & $469(76)$ & $323(75)$ & 0.71 \\
\hline Re-do & $150(24)$ & $106(25)$ & 0.70 \\
\hline \multicolumn{4}{|l|}{ Persistent AF, n(\%) } \\
\hline De-novo & $338(67)$ & $261(68)$ & 0.76 \\
\hline Re-do & 166(33) & $143(32)$ & 0.76 \\
\hline Procedure time, min, & $143 \pm 94$ & $140 \pm 91$ & 0.49 \\
\hline \multicolumn{4}{|l|}{ mean \pm SD } \\
\hline Fluoroscopy time, min, & $9.5 \pm 9$ & $9 \pm 7$ & 0.19 \\
\hline mean \pm SD & & & \\
\hline
\end{tabular}

Table 2: Procedural characteristics. The procedural characteristics of the DOAC $(n=$ $1123)$ and Warfarin $(n=761)$ group are shown in this table. Data are reported as percentage or mean \pm SD.

\begin{tabular}{|lccc|}
\hline $\mathrm{n}=1884$ & $\begin{array}{c}\text { DOAC } \\
(\mathrm{n}=1123)\end{array}$ & $\begin{array}{c}\text { WARFARIN } \\
(\mathrm{n}=761)\end{array}$ & $P$ value \\
\hline CVA/TIA $\mathrm{n}(\%)$ & $0(0)$ & $0(0)$ & 1 \\
\hline Cardiac Tamponade $\mathrm{n}(\%)$ & $19(1.7)$ & $8(1.1)$ & 0.28 \\
\hline Pseudoaneurysm $\mathrm{n}(\%)$ & $5(0.4)$ & $2(0.2)$ & 0.45 \\
\hline Haematoma $\mathrm{n}(\%)$ & $3(0.3)$ & $6(0.8)$ & 0.13 \\
\hline Blood Transfusion $\mathrm{n}(\%)$ & $3(0.3)$ & $1(0.1)$ & 0.36 \\
\hline Phrenic Nerve Palsy $\mathrm{n}(\%)$ & $6(0.5)$ & $1(0.2)$ & 0.29 \\
\hline
\end{tabular}

This article is protected by copyright. All rights reserved. 
Table 3: Procedural complications. The procedural complications of the DOAC $(n=1123)$ and Warfarin $(n=761)$ group are shown in this table. Data are reported as percentages. 